\title{
NIF Beam Shaping Masks
}

M.W.Bowers, M.A.Henesian,

\section{November 15, 2001}

Lawrence

Livermore

National

Laboratory

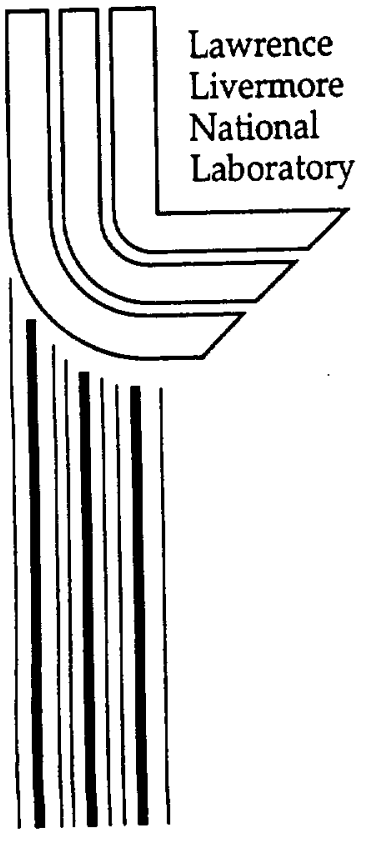




\section{DISCLAIMER}

This document was prepared as an account of work sponsored by an agency of the United States Government. Neither the United States Government nor the University of California nor any of their employees, makes any warranty, express or implied, or assumes any legal liability or responsibility for the accuracy, completeness, or usefulness of any information, apparatus, product, or process disclosed, or represents that its use would not infringe privately owned rights. Reference herein to any specific commercial product, process, or service by trade name, trademark, manufacturer, or otherwise, does not necessarily constitute or imply its endorsement, recommendation, or favoring by the United States Government or the University of California. The views and opinions of authors expressed herein do not necessarily state or reflect those of the United States Government or the University of California, and shall not be used for advertising or product endorsement purposes.

This work was performed under the auspices of the U. S. Department of Energy by the University of California, Lawrence Livermore National Laboratory under Contract No. W-7405-Eng-48.

This report has been reproduced

directly from the best available copy.

Available to DOE and DOE contractors from the

Office of Scientific and Technical Information

P.O. Box 62, Oak Ridge, TN 37831

Prices available from (423) 576-8401

http://apollo.osti.gov/bridge/

Available to the public from the

National Technical Information Service

U.S. Department of Commerce 5285 Port Royal Rd., Springfield, VA 22161

http://www.ntis.gov/

OR

Lawrence Livermore National Laboratory

Technical Information Department's Digital Library

http://www.Ilnl.gov/tid/Library.html 
To: Emmanuel Marquis

From: Mark Bowers

Subject: NIF beam shaping masks

Your question was not as simple to answer as I had first suspected. We were working through some issues with how the shapers/apodizers were being used and I did not want to give you incorrect information. They are designed to have somewhat high loss through the first pinhole and that was causing some confusion in the multipass amplifier. That issue has been straightened out and this information is correct. There are three attachments that will explain a little more about how the spatial shapers work. But the main point is that they are binary masks ( 0 or $100 \%$ transmission). The pattern of the $24 \times 24 \mu \mathrm{m}$ hole determines the overall transmitted spatial intensity. It is important to note that there is a lot of high frequency noise until the first spatial filter (for us it is $100 \mu \mathrm{rad}$ related to the $372 \mathrm{~mm}$ beam size). This first filter does take out almost all of the high frequency noise and leave a very nice beam. Of the three attachments, two explain how the apodizer works and one shows a binary image that we transfer onto a glass plate with Chromium.

We are using Sine Patterns

\section{Sine Patterns lle Pittsford, NY 14534 \\ Phone: (716) 385-1110 Fax: (716) 385-9528 \\ http://www.sinepatterns.com/}

with much success. They can create two kinds of masks for us. The first kind is on their standard substrate and without AR coatings. We then coat these with a sol-gel AR coating in-house. These take a 1-2 weeks for delivery. I don't have a good price on those. The other masks are on "NIF" qualified glass and have a hard AR coating under the Chromium. These are more expensive and take 20 weeks ARO. The price from them has been $\$ 1600$ (US) each.

Please feel free to contact me if you have more questions and again, sorry for the delay.

Best Regards,

Mark Bowers 


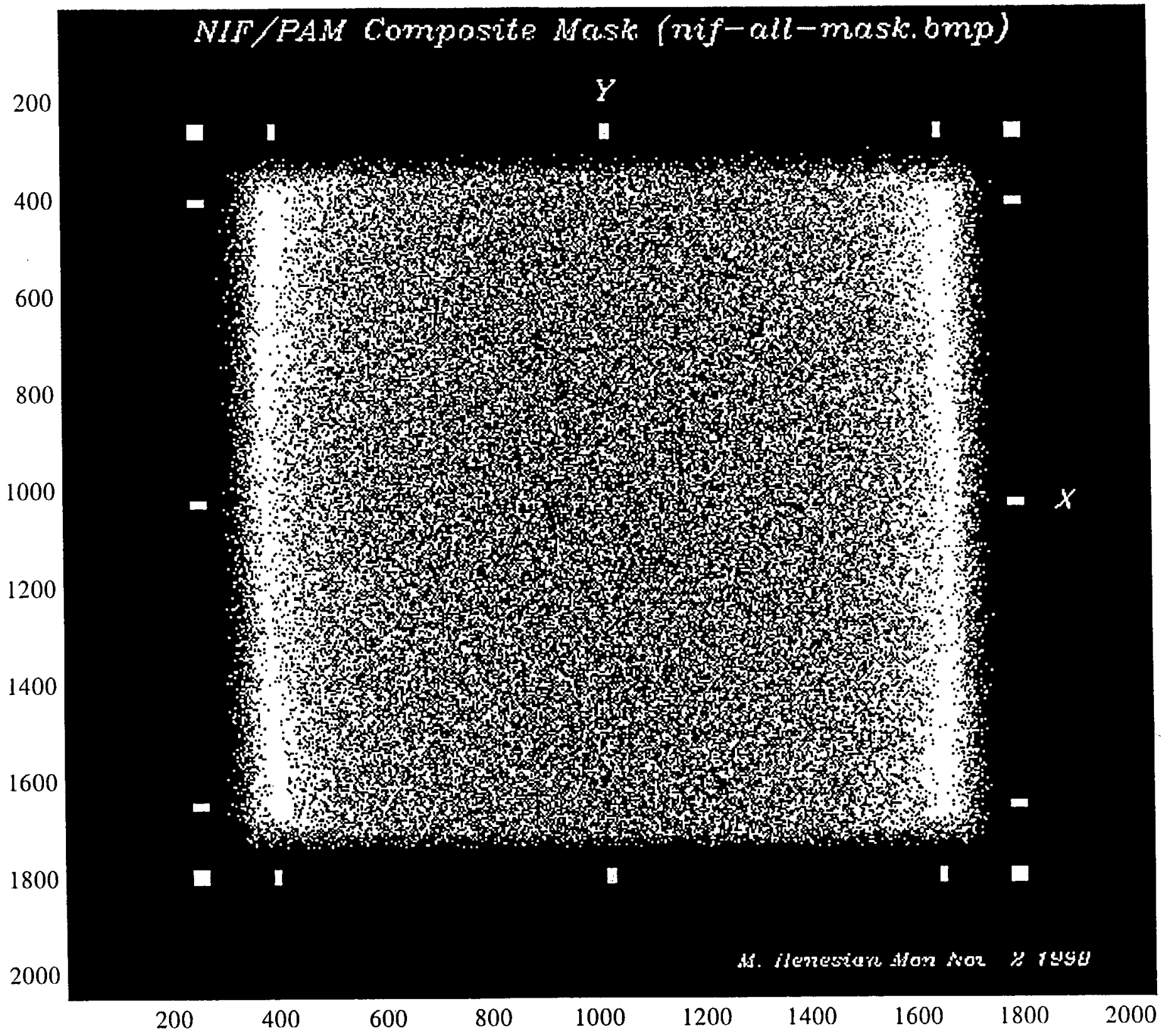


Inertial Confinement Fusion Program

ICF Laser Science and Technology

Laser Modeling and Optimization Group

Mail Station: $L-472$

Ext: 3-1504

June 7, 1999

LST-LMO-99-012

NIF-0025092

W.B.S. 1.3.1

To: $\quad$ Distribution

From: Mark A. Henesian

Subject: $\quad$ NIF beam shaping mask specifications

Summary: the enclosed e-mail describes the predicted transmission specifications on the four manufactured PAM/NIF spatial shaping masks (described below). Color contour and surface plots are attached for the analytic transmission profile of each mask.

Date: Sat, 05 Jun 1999 16:44:53 -0700

To: hermann1@Ilnl.gov, crane1@IInl.gov, moran5@llnl.gov, martinez31@linl.gov, skulina1@llnl.gov

From: "Mark A. Henesian" <henesian1 @llnl.gov>

Subject: NIF Beam Shaping Mask Specifications

Cc: hackel2@llnl.gov, aikens1@llnl.gov, sacks1@llnl.gov, wwilliams@llnl.gov, lawson3@Ilnl.gov, henesian1@llnl.gov

\section{Shaping description and mask transmission specifications:}

Attached are color contour and surface profile pictures of the four spatial shaping masks required for PAM testing. The masks have been described previously (e-mail sent Jan 7, 1999 and document NIF-0023967 dated May 26, 1999). These pictures were derived from the binary patterns and represent the transmission after spatial filtering in the PAM. The masks have been calculated for a $32 \times 32 \mathrm{~mm}$ beam footprint, which allows some beam size margin, and also the ability to "tune" the shape by small side-ways translation in the PAM shaping module. Note that each mask requires a serrated apodizer to define the beam edge - each mask used alone will roughly apodize the elliptical gaussian regen beam at the $-31 \times 31 \mathrm{~mm}$ size.

The binary chrome-on-glass patterns were transferred to Photosciences in Torrance, CA., who manufactured two (?) of each design. The binary patterns (in PC 8-bit binary file format) were each 4 MB in size and were $2048 \times 2048$ pixels in width and height, each pixel was $24 \times 24$ microns. The binary patterns were designed to compensate for the diffraction loss over and above the obscuration loss of the chrome pixels. These new spatial shaper designs, when hard AR coated, should get us very close to the theoretical energy transmissions (compiled below).

The masks are described below with center and overall transmission values for a "flat" $30 \times 30 \mathrm{~mm}$ test beam used with the TAB 10 error function serrated beam edge-apodizer.

1.) "ellipt-mask": used to convert $39 \times 33 \mathrm{~mm}$ fwl/e regen beam to a flat shape at the shaping module relay plane. Center reference transmission $=26.0 \%$, Energy transmission $=40.6 \%$, Peak/Center transmission ratio $=2.856$. 
2.) "pam-mask": used to convert $39 \times 33 \mathrm{~mm}$ fw l/e regen beam to a flat output shape at the RP8 relay plane of the 4-pass amplifier over the entire operating range ( 1 to $16+$ joules). Center reference transmission $=66.1 \%$, Energy transmission $=75.9 \%$, Peak/Center transmission ratio $=1.417$.

3.) "nif-mask": used in conjunction with "pam-mask" to convert the $39 \times 33 \mathrm{~mm}$ fwl/e regen beam to a flat output shape at the NIF output relay plane (KDP plane) over the full range of NIF energies for the SSMP and ICF missions. Output shape is "flat" at low energy (1-kJ/beamline) and slightly convex at high energy $(20 \mathrm{~kJ} / \mathrm{beamline})$. Center reference transmission $=18.6 \%$, Energy transmission $=27.5 \%$, Peak/Center transmssion ratio $=3.074$. When used with "pam-mask" energy transmisssion is approx. $18.1 \%$.

4.) "nif-all-mask": a high efficiency composite profile used individually to convert the $39 \times 33 \mathrm{~mm}$ fwl/e regen beam to a flat output shape at the NIF output relay plane (KDP plane) over the full range of NIF energies for the SSMP and ICF missions. Same output shape as using "pam-mask" and "nif-mask" together. Center reference transmission $=13.7 \%$, Energy transmission $=23.7 \%$, Peak/Center transmission ratio $=4.250$.

Performance Summary (when masks are used with $39 \times 33 \mathrm{~mm}$ elliptical gaussian regenerative amplifier beam):

1.) For "flat" beam at shaping module output using the "ellipt-mask", the energy transmission of the mask is predicted to be $\underline{20.1 \%}$.

2.) For "flat" output at RP8/RP10 using the "pam-mask", the energy transmission of the mask is predicted to be $\underline{47.3 \%}$.

3.) For "flat" output at NIF KDP plane using the "nif-all-mask", the energy transmission of the mask is predicted to be $\underline{11.4 \%}$

4.) Fot "flat" output at NIF KDP plane using the combination of the "pam-mask" + "nif-mask", the overall energy transmission of the combination is predicted to be $10.3 \%$

These transmissions assume that the shaping masks are used with the TAB 10 serrated apodizer. With the "softer" edged TAB 11 and TAB 12 designs (NIF-0015283 document, dated October 11, 1998) the overall shaping efficiency will be reduced.

Mark Henesian 6/5/99 

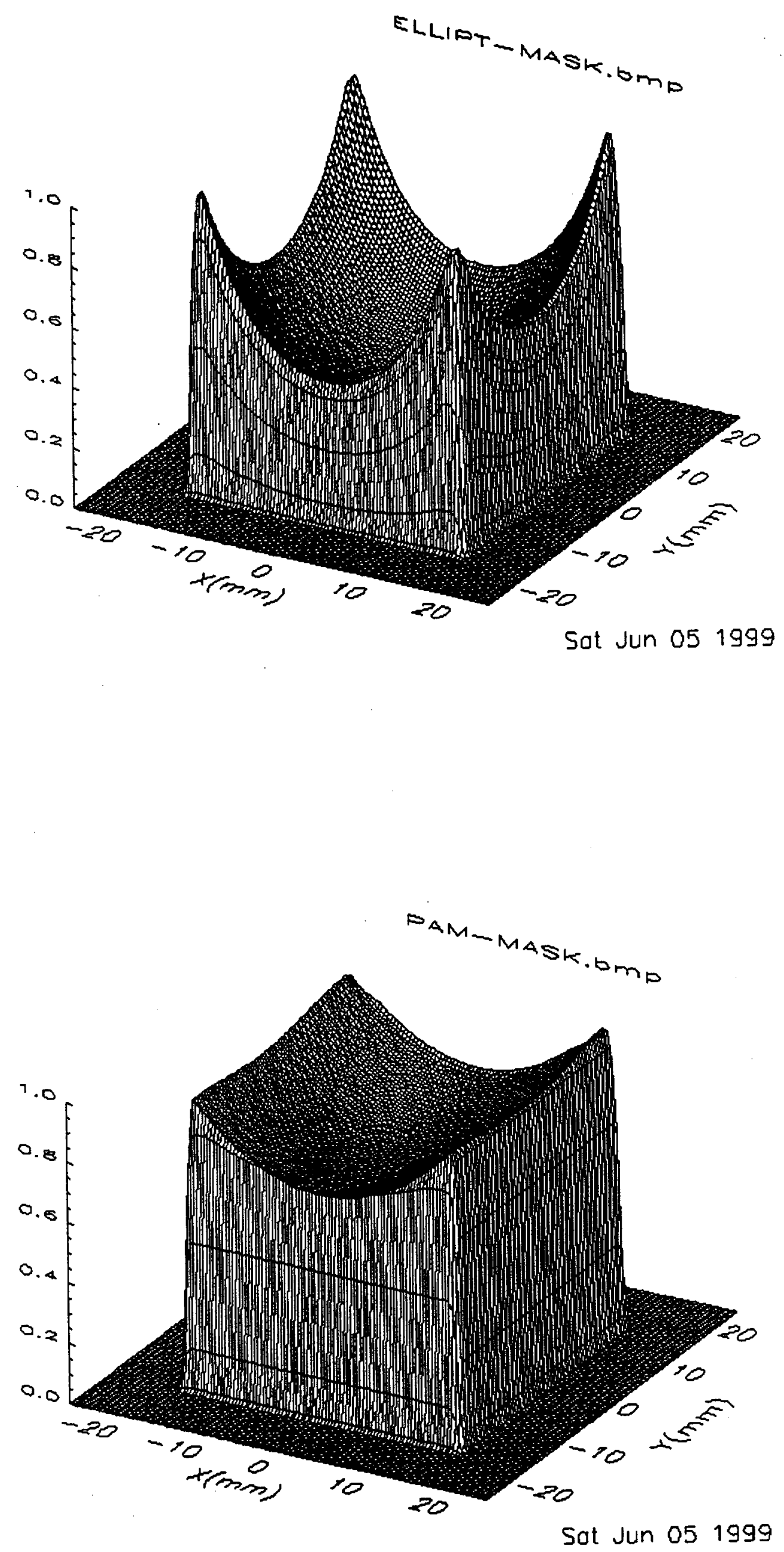

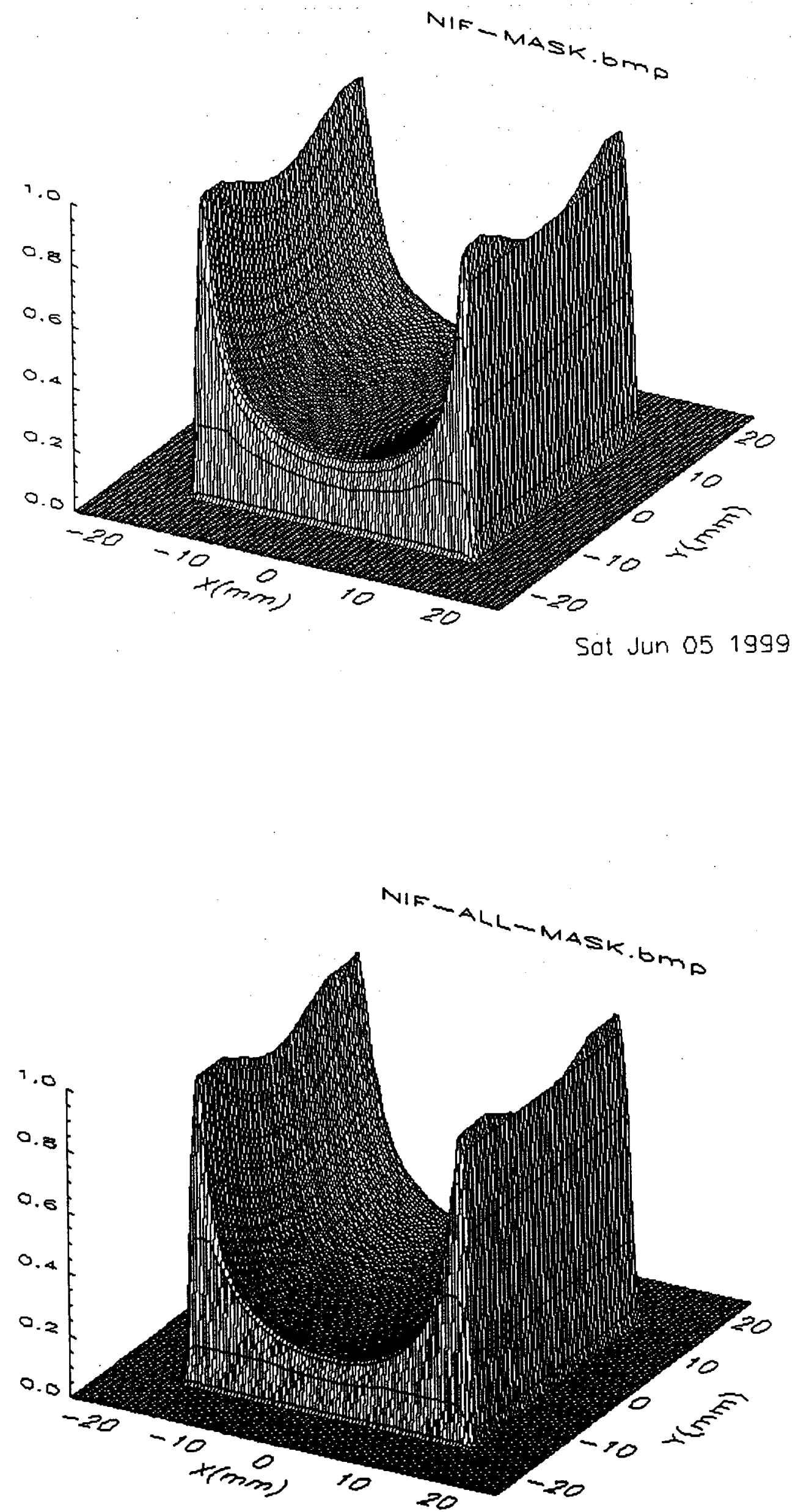

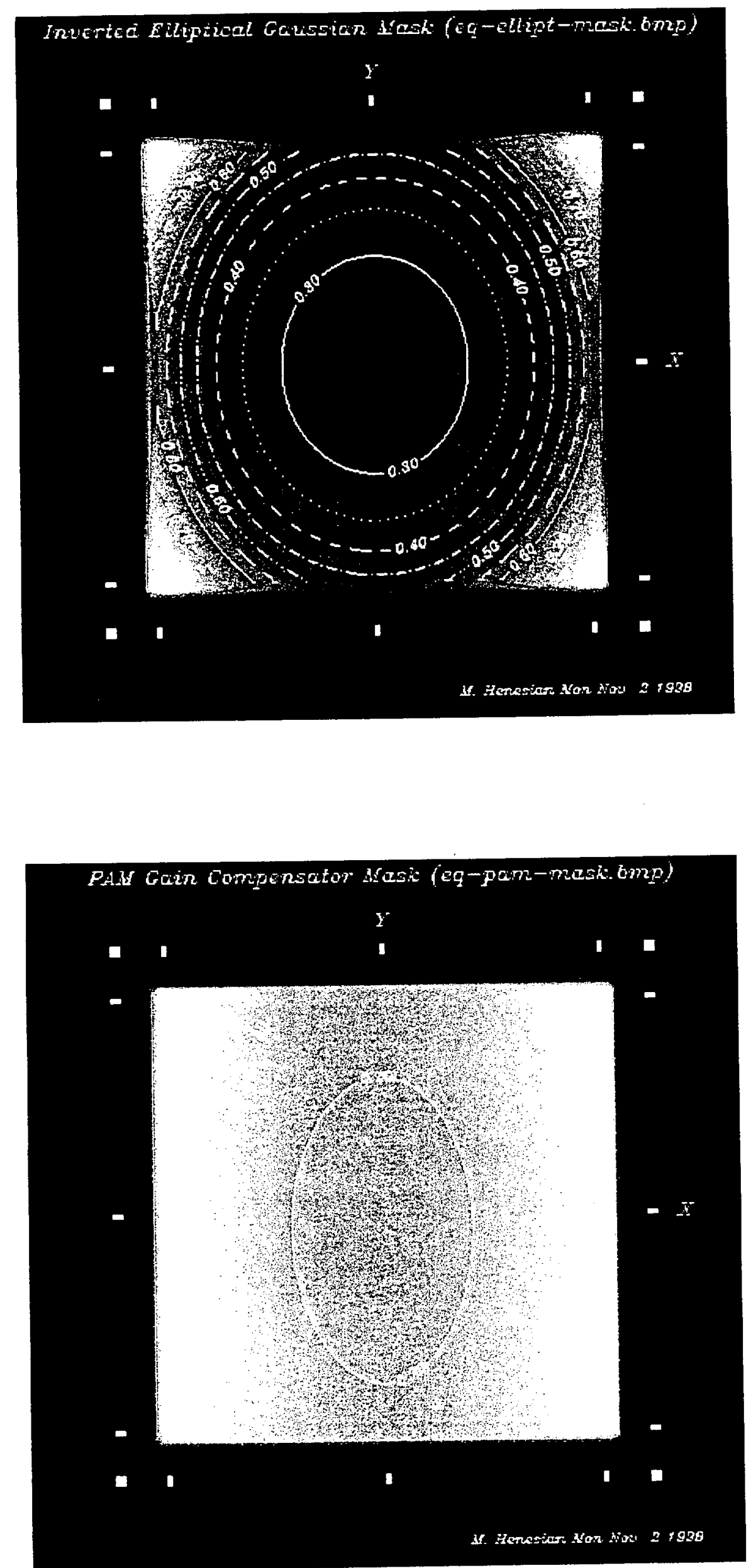

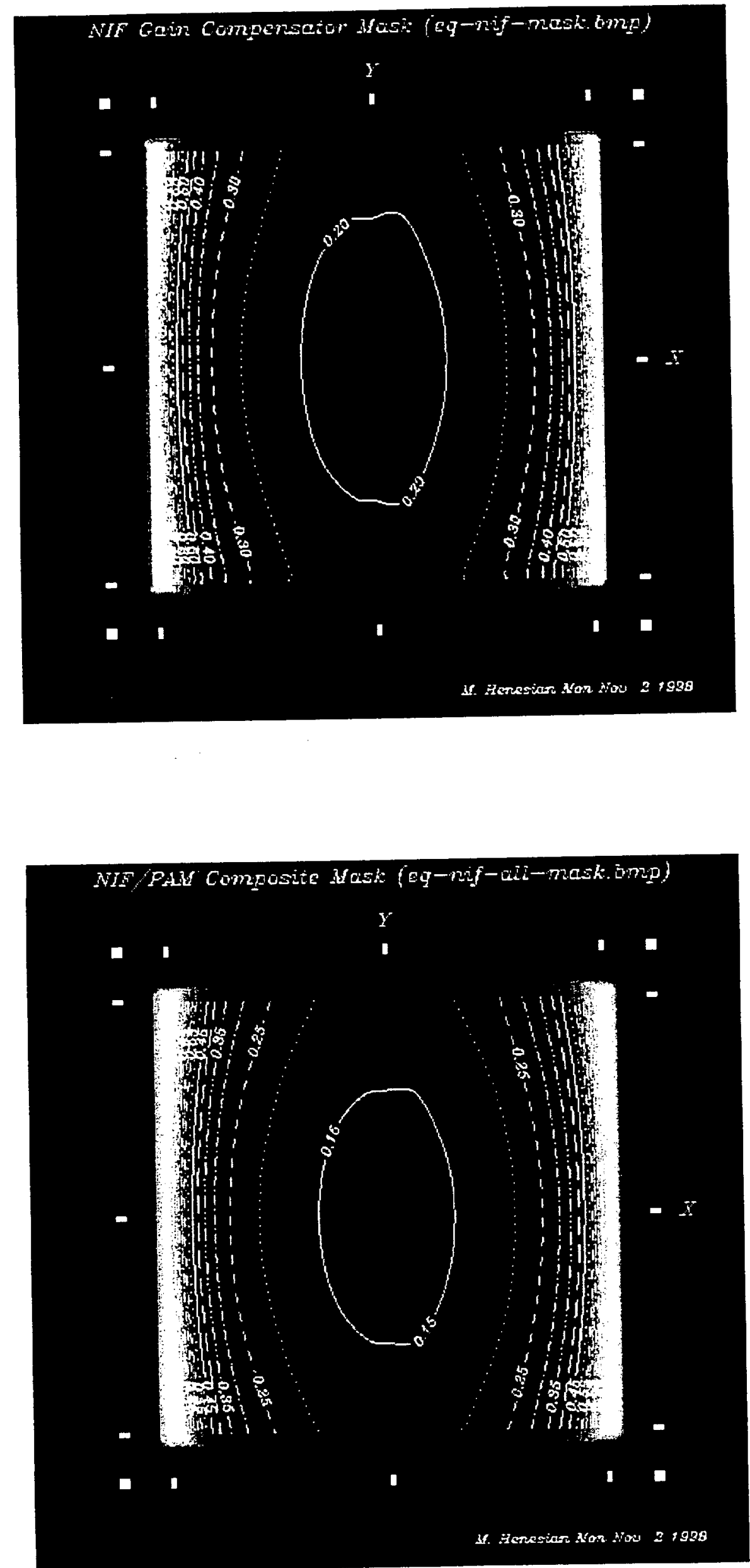
Inertial Confinement Fusion Program

ICF Laser Science and Technology

Laser Modeling and Optimization Group

Mail Station: $L-472$

Ext: $3-1504$

May 26, 1999

LST-LMO-99-011

NIF-0023967

W.B.S. 1.3.1

To: $\quad$ Distribution

From: Mark A. Henesian

Subject: $\quad$ NIF and PAM beam shaping mask descriptions

Summary: the following mask specifications need to be incorporated

in an updated AAA-97-106261 drawing package, entitled "OPG/Injection

Laser System Beam Shaping Filters".

Date: Thu, 07 Jan 1999 12:52:51 -0800

To: skulinal@llnl.gov, cranel@llnl.gov, hermann1@llnl.gov, moran5@llnl.gov, martinez31@llnl.gov

From: "Mark A. Henesian" <henesian1 @llnl.gov>

Subject: Beam Shaping Mask Nomenclature/Summary

Cc: english2@llnl.gov, aikens1@llnl.gov, henesian1@llnl.gov, trenholme1@llnl.gov

\section{NIF/PAM Shaping Mask Summary:}

The masks are designed for a $30 \times 30 \mathrm{~mm}$ beam in the PAM, but are slightly oversize (not much) to allow some beam size margin, and to "tune" the shape somewhat by small side-ways translation in the PAM shaping module. Note that each mask requires a serrated apodizer in order to accurately define the beam edge - each mask used alone will only roughly apodize the elliptical gaussian regen beam. Alignment markings have been added to the mask patterns to allow accurate centering with crosshairs and X-Y orientation in the shaping module. The X-Y coordinates match those on the NIF 4-pass amplifier drawing.

The binary chrome-on-glass patterns are $2048 \times 2048$ pixels in width and height, each pixel is $24 \times 24$ microns. The patterns are designed to compensate for diffractive loss over and above the obscuration loss of the chrome (black) pixels, and should get us close to the theoretical energy transmissions possible when hard-AR coated.

Nomenclature:(as encoded on each mask)

1.) Inverted elliptical gaussian mask ("ellipt-mask.bmp"): used to convert the $39 \times 33 \mathrm{~mm}$ fwl/e regen beam to a flat output shape at the shaping module relay plane RPO. This mask is for test only - will not be used on NIF.

2.) PAM gain compensator mask ("pam-mask.bmp"): used to convert regen beam to a flat output shape at the RP8 relay plane of the 4-pass amplifier over the operating range (1 to $16+$ joules).

3.) NIF gain compensator mask ("nif-mask.bmp"): used in conjunction with "pam-mask" to convert regen beam to a flat output shape at the NIF output plane (KDP plane) over the full range of NIF energies for the SSMP and ICF missions. Output shape is "flat" at low energy ( $1-\mathrm{kJ} / \mathrm{beamline})$ and slightly convex at high energy ( $20 \mathrm{~kJ} / \mathrm{beamline})$. 
4.) NIF/PAM composite mask ("nif-all-mask.bmp"): a high efficiency composite profile used individually to convert the regen beam to a flat output shape at the NIF output relay plane (KDP plane) over the full range of NIF energies for the SSMP and ICF missions. Same output shape as using "pam-mask" and "nif-mask" together.

Efficiencies:

1.) "pam-mask": for "flat" output at RP8/RP10 the overall energy transmission from regen output (not including losses in regen transport optics and 20X Newport telescope lenses, diagnostics splitter, temporal pulse slicer, etc.) to the beam shaping module output plane is $47.25 \%$.

2.) "nif-all-mask": for "flat" output at NIF KDP plane, the overall energy transmission from regen output (not including losses above,etc.) to the beam shaping module output plane is $11.42 \%$

3.) "pam-mask" + "nif-mask": for "flat" output at NIF KDP plane, etc, efficiency is $10.28 \%$

The shaper efficiency estimates assume that the masks are used with the TAB 10 serrated apodizer. With the "softer" edged TAB $\|$ and TAB 12 designs the overall efficiency will be reduced. Used alone, the inverted elliptical gaussian mask will have an efficiency of $\sim 25.7 \%$. With the TAB 10 apodizer, the efficiency will drop to $20.1 \%$.

An updated PROP92 model for the PAM/ISP/PABTS system indicates that a regen energy at the shaper input of 4.5 $\mathrm{mJ}$ will be required to achieve a 16.0-J spatially shaped output at RP8, and $3.02 \mathrm{~J}$ at the NIF injection mirror. For $-20.6 \mathrm{~kJ}$ at the KDP plane, however, only $\sim 6$ Joules is required at RP8, and this requires $-1.2 \mathrm{~mJ}$ at the shaper input. The regen energies are based on a 5-cm Nova rod amplifier energy gain of 16.8/pass and without losses in the 4-pass cavity associated with the SSD grating. All numbers are subject to minor revision.

I'm particularly gratified that the initial PAM measurements are in agreement with my estimates,

Mark Henesian 1/7/99 
This work was performed under the auspices of the U.S. Department of Energy by the University of California, Lawrence Livermore National Laboratory under Contract No. W-7405-Eng-48. 